\title{
INCIDÊNCIA HIPOTIREOIDISMO CONGÊNITO EM CRIANÇAS NASCIDAS NOS HOSPITAIS PÚBLICOS DO OESTE PAULISTA
}

\author{
Franciele Cardoso Leite, Patricia Martins Luizari Escoboza
}

Hospital Regional de Presidente Prudente: Associação e Fraternidade São Francisco de Assis na Providência de Deus. e-mail: leite franciele@yahoo.com.br

\section{RESUMO}

Hipotiroidismo congênito é uma causa de retardo mental e apresenta sinais e sintomas inespecíficos. O diagnóstico e tratamento precoces são fundamentais para o desenvolvimento normal das crianças acometidas por esta alteração. Esse estudo teve o objetivo de avaliar a incidência da alteração nos testes de triagem dos hospitais públicos de Presidente Prudente, a média de idade do recém-nascido na ocasião da coleta dos exames e na confirmação do diagnóstico. Foi realizado um estudo retrospectivo do tipo transversal para avaliar os dados dos recém-nascidos que realizaram o "teste do pezinho" pelo sistema único de saúde em 2017. Dos 3.351 avaliados, 48 apresentaram elevação da concentração do Hormônio Estimulante da Tireoide (TSH) e 13 tiveram o diagnóstico de hipotiroidismo congênito confirmado. Foram $61,54 \%$ os que retornaram entre os dias 16 e 30 para confirmação do resultado e $23,07 \%$ aqueles que retornaram após 61 dias. 0 diagnóstico e tratamento precoces são fundamentais, pois possibilitam ações mitigadoras dos graves distúrbios cognitivos e comportamentais dos pacientes afetados.

Palavras-chave: triagem neonatal, hormônios tireóideos, hipotireoidismo congênito, recém-nascido, sistema único de saúde.

\section{INCIDENCE OF CONGENITAL HYPOTHYROIDISM IN CHILDREN BORN IN PUBLIC HOSPITALS IN WESTERN SÃO PAULO}

\section{ABSTRACT}

Congenital hypothyroidism is a cause of mental retardation and has nonspecific signs and symptoms. Early diagnosis and treatment are essential for the normal development of children affected by this disorder. The objective of this study was to evaluate the incidence of changes in the screening tests of public hospitals in Presidente Prudente, the average age of the newborn at the time of collection of tests and confirmation of the diagnosis. A retrospective cross-sectional study was carried out to assess the data of newborns who underwent the "heel prick test" by the unified health system in 2017. Of the 3,351 evaluated, 48 had elevated levels of Thyroid Stimulating Hormone (TSH), 13 had the diagnosis confirmed congenital hypothyroidism. It was $61.54 \%$ the children that returned between days 16 and 30 to confirm the result and $23.07 \%$ that returned after 61 days. Early diagnosis and treatment is essential, as they enable mitigating actions for the serious cognitive and behavioral disorders of affected patients.
\end{abstract}

Keyword: neonatal screening, thyroid hormones, congenital hypothyroidism, newborn, unified health system.

\section{INTRODUÇÃO}

O Hipotireoidismo Congênito é uma emergência pediátrica causada pela incapacidade da glândula tireoide do recém-nascido em produzir quantidades adequadas de hormônios 
tireoideanos, que resulta numa redução generalizada dos processos metabólicos ${ }^{1}$.

O Hipotireoidismo Congênito é uma das causas mais comuns de retardo mental evitável no mundo, apresentando sinais e sintomas bastante inespecíficos, de forma que em apenas $5 \%$ das crianças afetadas é possível o diagnóstico através do exame clínico nos primeiros dias de $v^{v i d a}{ }^{2}$. O diagnóstico e o tratamento precoces são fundamentais para o crescimento e desenvolvimento intelectual normal dessas crianças ${ }^{3}$.

O hormônio tireoidiano é essencial para o desenvolvimento do sistema nervoso central, e a deficiência desses hormônios na vida fetal e no recém-nascido mantém a imaturidade deste tecido, leva à hipoplasia dos neurônios corticais, atraso da mielinização e redução da vascularização. Se a reposição hormonal não se fizer logo após o nascimento, essas lesões tornarse-ão irreversíveis, com prejuízo do desenvolvimento neuropsicomotor ${ }^{4}$ A diminuição drástica dos hormonios tireoideanos observada em crianças com Hipotireoidismo Congênito, pode gerar danos importantes e sequelas irreversiveis ao Sistema Nervoso Central $^{5}$, comprometendo o desenvolvimento intelectual destes individuos ${ }^{6}$. Desta maneira o diagnóstico precoce da Hipotireoidismo Congênito é fundamental, pois possibilita o tratamento correto e prevenção das repercussões neurológicas ${ }^{4}$. A maioria das crianças afetadas apresenta sinais e sintomas bastante inespecíficos, o que dificulta o diagnóstico clínico nos neonatos, ademais, uma parcela importante dos pacientes apresentam-se normal ao nascimento ${ }^{7}$. Com início, na década de 80, dos programas de triagem neonatal, nos quais, exames baseados em dosagens hormonais em sangue seco colhido em papel de filtro, começaram a ser aplicados e se mostraram essenciais e eficazes para a resolução destas questões ${ }^{8}$.

A estratégia de diagnóstico do Hipotireoidismo Congênito, incluindo os parâmetros utilizados e os seus valores de referência, têm-se alterado ao longo dos anos. $\mathrm{Na}$ maioria dos países da Europa, no Japão, Canadá e em parte dos Estados Unidos utiliza-se a determinação do Hormônio Estimulante da Tireóide (TSH) como parâmetro inicial e o T4 (tiroxina) como exame secundário ${ }^{9}$. Inicialmente a colheita de sangue era efetuada entre $\circ 6^{\circ}$ e o $10^{\circ}$ dias de vida, porem, com a permanência das puérperas no hospital mais curta, a colheita tem sido efetuada entre $\circ 3^{\circ}$ e $6^{\circ}$ dia de vida e ocasionalmente mais cedo, o que tem aumentado o número de falsos positivos ${ }^{10}$. No Brasil a Triagem Neonatal para Hipotireoidismo Congênito é realizada pela dosagem de TSH [no caso de um recém-nascido (RN) afetado, este hormônio está elevado] e/ou pela dosagem de T4 (no caso de um RN afetado, este hormônio está diminuído). As dosagens são realizadas em sangue colhido em papel filtro, sendo considerados resultados alterados aqueles $\geq 10$ $\mu \mathrm{UI} / \mathrm{mL}$. Valores maiores do que este, sugerem o diagnóstico de Hipotireoidismo Congênito e demandam uma avaliação clínica imediata do recém-nascido e confirmação diagnóstica através da dosagem sérica de TSH e T4 ${ }^{1,11}$.

A realização dos exames nesta sequência permite a detecção da maioria dos casos. Estudo recente sugere que a utilização do ponto de corte de TSH de $10 \mu \mathrm{UI} / \mathrm{L}$ no Teste do Pezinho, aumenta sensivelmente a identificação de pacientes com hipotireoidismo congênito ${ }^{1}$.

Os sinais mais precoces são: icterícia prolongada ou recorrente, atraso na queda do funículo umbilical e hérnia umbilical, dificuldade alimentar, ganho de peso insuficiente, respiração ruidosa, congestão nasal, distúrbios respiratórios, obstipação, letargia, pele seca, fria, pálida e com livedo reticularis. Contudo, esses sinais e sintomas nem sempre se apresentam de modo evidente, podendo-se perder um tempo precioso para o início do tratamento. Daí a importância dos testes laboratoriais em berçário ${ }^{4}$.

A finalidade do Teste Triagem Neonatal ("Teste do Pezinho") é o rastreamento neonatal de crianças portadoras de doenças que devem ser diagnosticadas e tratadas o mais precocemente possível, como o Hipotireoidismo Congênito e a Fenilcetonúria (PKU), Anemia Falciforme e de outras hemoglobinopatias e da Fibrose Cística $(\mathrm{FC})$, é oferecido gratuitamente à população, e tem como objetivo identificar distúrbios e doenças no recém-nascido em tempo oportuno para intervenção adequada, garantindo tratamento e acompanhamento contínuo, com vistas a reduzir a morbimortalidade e melhorar a qualidade de vida das pessoas com doenças previstas na política $^{11}$. É realizado em laboratório a partir de amostras de sangue retiradas do calcanhar do recém-nascido e colhidas em papel filtro. Somente no estado de Minas Gerais foram

Colloq Vitae 2021 jan-abr; 13(1): 1-6.

Artigo Open Access sob uma licença CC BY-NC-ND (http://creativecommons.org/licenses/by-nc-nd/4.0/). 
triados quase 6 milhões de recém nascidos entre os anos de 1994 e $2018^{12}$.

A Organização Mundial de Saúde (OMS) preconiza desde a década de 60 a programas de Triagem Neonatal, para a prevenção de deficiência mental e agravos à saúde, e destaca sua importância na prevenção de problemas como retardo mental ou mesmo o óbito do recém-nascido e recomenda sua implementação, especialmente nos países em desenvolvimento ${ }^{13,14}$. Entre os benefícios da triagem neonatal está a detecção de doenças graves e tratáveis antes do aparecimento dos sintomas ${ }^{14}$.

A incidência de Hipotireoidismo Congênito é quatro a cinco vezes maior do que a da fenilcetonúria, para a qual os programas de triagem foram originalmente desenvolvidos. É de aproximadamente 1:3.000 a 1:4.000 recémnascidos vivos, sendo maior em hispânicos e menor em negros. Algumas variações de incidência entre diferentes regiões geográficas parecem estar mais relacionadas com a deficiência de iodo do que com as características étnicas populacionais. Há prevalência do sexo feminino em relação ao masculino (2:1), e há risco aumentado em crianças com síndrome de Down $^{15}$.

O Hipotireoidismo Congênito é considerado uma urgência pediátrica, podendo ocorrer consequências graves quando não recebe tratamento oportuno. O diagnóstico e tratamento precoce são fundamentais para o desenvolvimento intelectual das crianças afetadas. $O$ conhecimento referente à prevalência das doenças neonatais favorece a elaboração e o aprimoramento das políticas públicas voltadas para a saúde da criança, minimiza gastos em serviços de alta complexidade e contribui para a redução da taxa de mortalidade infantil ${ }^{16}$.

O principal objetivo deste trabalho foi avaliar a incidência da alteração do TSH nos testes de triagem neonatal dos hospitais públicos do município de Presidente Prudente/SP que foram encaminhados no período entre janeiro e dezembro de 2017. Nesta investigação foram determinar o tempo transcorrido entre a primeira coleta e a confirmação do diagnóstico para o início do tratamento, além de analisar a incidência de neonatos com hipotireoidismo congênito.

\section{METODOLOGIA}

Foi realizado um estudo retrospectivo do tipo transversal baseado no banco de dados do serviço de referência de triagem neonatal de um hospital terciário de Presidente Prudente, São Paulo, Brasil, tendo como amostra todos os recém-nascidos que realizaram 0 teste de triagem neonatal na rede de coleta conveniada ao sistema único de saúde, no período de janeiro a dezembro de 2017. De acordo com normas estabelecidas pelo Ministério da Saúde ${ }^{1}$, é responsabilidade do serviço de referência em triagem neonatal a identificação e capacitação dos postos de coleta, a distribuição de material necessário, bem como o treinamento $\mathrm{e}$ conscientização dos recursos humanos administrativos. Os dados foram obtidos por médico residente de pediatria do hospital, a partir de relatórios obtidos no banco de dados do serviço de referência. Quanto às questões relacionadas à organização do serviço, foram pesquisados dados referentes à cobertura do programa de triagem pela rede pública da cidade de Presidente Prudente, sendo incluído nesse estudo o número estimado de crianças nascidas vivas na cidade de Presidente Prudente, número de crianças testadas pelo serviço, idade das crianças na primeira coleta, tempo transcorrido entre a primeira coleta e a entrega dos resultados e a incidência da patologia testada. Foram utilizados dados referentes ao número de nascidos vivos do ano de 2017, obtidos junto ao banco de dados dos hospitais públicos que foram referenciados ao hospital terciário de Presidente Prudente. A partir desses dados, foram calculados: o percentual, valor máximo e mínimo de algumas variáveis. A realização do trabalho foi aprovada pelo Comitê de Ética em Pesquisa da Universidade do Oeste Paulista - UNOESTE, sob o protocolo CAAE $\mathrm{n}^{\circ}$ 17607419.7.0000.5515, parecer $n^{\circ}$ 3.701.714.

As análises estatísticas descritivas foram realizadas utilizando o pacote estatístico BioEstat 5.

\section{RESULTADOS}

No ano de 2017, os nascidos vivos em nos hospitais públicos Presidente Prudente totalizaram 3.351 crianças, deste total 2.292 crianças nasceram num hospital estadual e 1059 num hospital terciário

Dos 3.351 nascidos vivos, 1.184 crianças eram filhos pais residentes no município de Presidente Prudente. Destes, 48 crianças 
apresentaram alterações nos níveis do TSH através do teste de triagem neonatal (teste do pezinho) e foram encaminhados ao serviço de neonatologia do Hospital Regional de Presidente Prudente para confirmação por dosagem sérica, o que representa uma incidência de $4,05 \%$ de exames alterados.

Apenas 13 pacientes encaminhados para confirmação dos resultados da dosagem de TSH tiveram as alterações confirmadas, os dados obtidos são apresentados abaixo (Tabela 1).

Tabela 1. Número de encaminhados, confirmados e a faixa de idade dos pacientes para confirmação do resultado do TSH.

\begin{tabular}{ccccc}
\hline $\begin{array}{c}\text { Encaminhados } \\
\text { para } \\
\text { confirmação }\end{array}$ & $\begin{array}{c}\text { Resultados } \\
\text { com }\end{array}$ & \multicolumn{2}{c}{$\begin{array}{c}\text { Idade dos } \\
\text { pacientes na } \\
\text { alteração } \\
\text { confirmada }\end{array}$} & \multicolumn{2}{c}{$\begin{array}{c}\text { ocasião do } \\
\text { retorno (dias) }\end{array}$} \\
\cline { 3 - 5 } & & $15<$ & $31<$ & $61<$ \\
& & $>30$ & $>60$ & $>90$ \\
\hline 48 & 13 & 8 & 2 & 3 \\
\hline
\end{tabular}

É possível observar que $27,08 \%$ dos pacientes encaminhados para confirmação de resultados tiveram o diagnóstico, e estes pacientes foram encaminhados para a sequência do tratamento com o médico endocrinologista. A maioria dos pacientes ( $n=8$, ou $61,54 \%$ ) retornaram entre os dias 16 e 30 após a dosagem inicial para confirmação do resultado, apenas 3 $(23,07 \%)$ retornaram após 61 dias. Quando consideramos o total de nascidos vivos, a prevalência de exames alterados após confirmação por dosagem sérica é de 1,09\% (13 neonatos).

\section{DISCUSSÃO}

Os resultados obtidos neste estudo não corroboram com a incidência no Brasil, que é de aproximadamente um caso para cada 2.595 a 4.795 nascidos vivos, no estado de São Paulo a incidência é de em um caso para cada 3.500 nascidos vivos ${ }^{17}$. Nas regiões não deficientes em iodo (como no Brasil), as principais causas de hipotiroidismo congênito são ectopia tiroidiana (em torno de $60 \%$ ), agenesia tiroidiana (em torno de $15 \%$ ) e deficiência na síntese hormonal (em torno de $15 \%$ ). Os casos de hipotiroidismo congênito central são mais raros, ocorrendo em cerca de 1:25.000-1:100.000 nascidos vivos ${ }^{16}$. Em Minas Gerais nos anos de 2000 e 2006 foram triadas 1.874 .055 crianças, um total de $99 \%$ de cobertura dos municípios da cidade, 464 crianças $(0,025 \%)$ foram diagnosticadas com Hipotireoidismo Congênito ${ }^{15}$. 0 hipotiroidismo congênito tem incidência variável, ocorrendo de 1:1.800 a 1:10.000 nascidos vivos na Grécia e na França, respectivamente ${ }^{16}$.

Em crianças a termo, com mais de 48 horas de vida, e valores de TSH neonatal menores que $10 \mu \mathrm{UI} / \mathrm{L}$ no sangue total, nenhum seguimento é realizado. Resultados de TSH entre 10 e $20 \mu \mathrm{UI} / \mathrm{L}$ determinam a solicitação de uma segunda amostra do calcanhar e, na maioria das vezes, este segundo resultado virá normal. Entretanto, quando o resultado do TSH neonatal for maior que $20 \mu \mathrm{UI} / \mathrm{L}$, solicita-se que a criança compareça para consulta clínica e realize testes de função tireoidiana em amostras de soro. A maioria das crianças com valores de TSH neonatal maior que $20 \mu \mathrm{UI} / \mathrm{L}$ apresentará a doença ${ }^{17,18}$.

Nosso estudo evidenciou $61,54 \%$ iniciou tratamento até primeiro mês de vida, corroborando com estudo de Pezzuti et al. ${ }^{15}$, durante o período estudado, $53 \%$ das crianças iniciaram o tratamento ainda no primeiro mês de vida, mas a maioria com mais de 15 dias, sendo a meta de acordo com o estudo de Regina ${ }^{18}$, a idade ideal para início do tratamento, deve ser no máximo até 14 dias, pois após essa idade, nos casos de Hipotireoidismo Congênito por atireose e disormonogêne grave, já poderá ocorrer algum dano cerebral. Quando o tratamento é iniciado precocemente, 0 impacto negativo de Hipotireoidismo Congênito grave desaparece.

A prevalência encontrada no Serviço de Referência em Triagem Neonatal da UNICAMP é de uma criança detectada a cada 3.500 nascidos vivos $^{19}$.

Alguns casos de mais raros de Hipotireoidismo Congênito podem não ser diagnosticado, como o caso do Hipotiroidismo Pituitário Hipotalâmico (aumento tardio do TSH), O mais indicado nesse caso é a realização de exames de ultrassonografia ou cintilografia com captação tireóidea de iodo radioativo, pois na maioria das vezes $85 \%$ a origem está na própria glândula tireoide ${ }^{11}$.

Muitos estudos de bases genéticas tentam elucidar os mecanismos moleculares relacionados à etiologia do Hipotireoidismo Congênito para aprimorar o diagnóstico e o aconselhamento genético. A evolução adequada dos pacientes com Hipotireoidismo Congênito depende do início precoce do tratamento, idealmente até 14 dias de vida, com dose de

Colloq Vitae 2021 jan-abr; 13(1): 1-6.

Artigo Open Access sob uma licença CC BY-NC-ND (http://creativecommons.org/licenses/by-nc-nd/4.0/). 
levotiroxina que normalize rapidamente os níveis hormonais (10 a $15 \mu \mathrm{g} / \mathrm{Kg} /$ dia no recém-nascido), e do acompanhamento regular para garantir que os níveis de hormônios tireoidianos estejam sempre na faixa normal. Não basta fazer o diagnóstico precoce e correto, é necessário garantir um tratamento adequado e contínuo para assegurar os melhores prognósticos possíveis tanto no desempenho escolar como na qualidade de vida ${ }^{15,20}$.

O tratamento ou diagnóstico precoce elimina os graves distúrbios cognitivos e comportamentais, sendo a desvantagem que ocorre com o diagnóstico tardio, é bem reconhecido que alguns pacientes tratados precocemente ainda apresentam déficit do quociente de inteligência (QI) e/ou de comportamento, problemas de atenção e atenção, bem como sutis motores, linguagem, e comprometimento visuo-espacial. Esses problemas são parcialmente relacionados à gravidade da doença, a julgar pelo T4 inicial, idade óssea retardo e sintomas clínicos no diagnóstico fatores pós-natais também afetam o resultado cognitivo, incluindo atraso no início do tratamento, a adequação e o cumprimento de tratamento, especialmente nos primeiros 3 anos e fatores socioeconômicos ${ }^{23}$.

Devido a importância do diagnóstico de Hipotireoidismo Congênito, se faz necessário, no sistema único de saúde, o comprometimento das políticas públicas voltadas a realizar os testes de triagem neonatal e se necessário os testes confirmatórios, em menor tempo possível, se justifica, uma vez que quando não diagnosticadas e tratadas precocemente, crianças com hipotireoidismo congênito apresentam desenvolvimento mental e crescimento seriamente afetados, sendo que o comprometimento da capacidade intelectual podendo ser irreversível. Um dos principais fatores para o prognóstico depende, fundamentalmente, do tempo decorrido para instituição do tratamento, sendo uma doença passivel de tratamento, e com a identificação de fatores de risco e diagnosticada em seu estágio inicial, realizando assim o encaminhamento ágil e adequado para o atendimento especializado dão à Atenção Básica um caráter essencial para um melhor resultado terapêutico e prognóstico dos casos.

\section{CONFLITO DE INTERESSES}

Os autores declaram não haver qualquer potencial conflito de interesse que possa interferir na imparcialidade deste trabalho científico.

\section{REFERÊNCIAS}

1. Portaria $\mathrm{n}^{\circ}$ 56, de 23 de abril de 2010. Ministério da Saúde, Brasili. 2010. Acesso em: 10 mai 2020. Disponível em: http://www.bvsms.saude.gov.br

2. LaFranchi CS, Dussault JH, Fisher DA, Foley TP, Mitchell M. Newborn screening academy of recommended for congenital hypothyroidism: guidelines development. Am Acad Pediatr. 1993;91(6):1203-9.

3. Fisher DA. The importance of early management in optimizing $I Q$ in infants with congenital hypothyroidism. J Pediatr. 2000;136(3):273-4. DOI: https://doi.org/10.1067/mpd.2000.104286

4. Setian N. Hypothyroidism in children: diagnosis and treatment. J Pediatr. 2007;83(5):209-16. DOI: https//doi.org/10.2223/JPED.1716

5. Cao X-Y, Jiang X-M, Dou Z-H, Rakeman MA, Zhang $M-L, O^{\prime}$ Donnell $K$, et al. Timing of vulnerability of the brain to lodine deficiency in endemic cretinism. N Engl J Med. 1994;331(26):1739-44. DOI: https://doi.org/10.1056/NEJM199412293312603

6. Porterfield SP, Hendrich CE. The role of thyroid hormones in prenatal and neonatal neurological development - current perspectives. Endocr Rev. 1993;14(1):94-106.

DOI: https://doi.org/10.1210/er.14.1.94

7. Rodrigues AL, Carvalho A, Duarte $C P$, César R, Anselmo J. Hipotiroidismo congénito. Rev Port Endocrinol Diabetes e Metab. 2014;7(1):28-35. DOI:

https://doi.org/10.1016/i.rpedm.2014.01.001

8. Rapaport R. congenital hypothyroidism: expanding the spectrum. J Pediatr. 2000;136(1):10-2. DOI: https://doi.org/10.1016/S0022-3476(00)90041-6

9. Rose SR, Brown RS, Foley T, Kaplowitz PB, Kaye $\mathrm{Cl}$, Sundararajan $\mathrm{S}$, et al. Update of newborn

Colloq Vitae 2021 jan-abr; 13(1): 1-6.

Artigo Open Access sob uma licença CC BY-NC-ND (http://creativecommons.org/licenses/by-nc-nd/4.0/). 
screening and therapy for congenital hypothyroidism. Pediatrics. 2006;117(6):2290303. DOI: https://doi.org/10.1542/peds.2006$\underline{0915}$

10. Rapaport R. Congenital hypothyroidism: an evolving common clinical conundrum. J Clin Endocrinol Metab. 2010;95(9):4223-5. DOI: https://doi.org/10.1210/jc.2010-1711

11. Triagem Neonatal Biológica: Manual Técnico. Ministério da Saúde, Brasil. 2016.

12. Programa de triagem neonatal de minas gerais - NUPAD. Acesso em: 10 mai 2020. Disponível em: https://www.nupad.medicina.ufmg.br/programae-acoes/programa-de-triagem-neonatal-deminas-gerais/

13. OMS. Manual de normas técnicas e rotinas operacionais do programa nacional de triagem neonatal. 2002. 90p.

14. Leatilde LL, Aguiar MJB. Newborn screening: What pediatricians should know. J Pediatr. 2008;84(7):80-90.

DOI: https://doi.org/10.2223/JPED.1790

15. Pezzuti IL, de Lima PP, Dias VM. Congenital hypothyroidism: The clinical profile of affected newborns identified by the newborn screening program of the state of minas gerais, Brazil. J Pediatr. 2009;85(1):72-9. DOI: https://doi.org/10.2223/JPED.1863

16. Luz GS, Carvalho MD de B, Pelloso SM, Higarashi IH. Prevalência das doenças diagnosticadas pelo programa de triagem neonatal em Maringá, Paraná, Brasil: 2001-2006. Rev Gaúcha Enferm. 2008;29(3):446-53.

17. Cargnin KRN, dePaula LCP, Garcia LS, CollettSolberg PF, et al. Hipotireoidismo congênito: triagem neonatal. Soc Bras Pediatr. 2018;5:1-12.

18. Protocolo clínico e diretrizes terapêuticas hipotiroidismo congênito. Portaria SAS/MS $\mathrm{n}^{\circ}$ 1161, de 18 de novembro. Ministério da Saúde, Brasil. 2015.

19. Vidal AM, Dias DO, Martins ESM, Oliveira RS, Nascimento RMS, Correia MGS. A ingestão de alimentos funcionais e sua contribuição para a Colloq Vitae 2021 jan-abr; 13(1): 1-6.

Artigo Open Access sob uma licença CC BY-NC-ND (http://creativecommons.org/licenses/by-nc-nd/4.0/). diminuição da incidência de doenças. Cad Grad Ciên Biol Saúde. 2012;1(15):43-52.

20. Maciel LMZ, Kimura ET, Nogueira CR, Mazeto GMFS, Magalhães PKR, et al. Hipotireodismo congenito: recomendações do Departamento de Tireoide da Sociedade Brasileira de Endocrinologia e Metabologia. Arq Bras Endocrinol Metab. 2013;57(3):184-91. DOI: https://doi.org/10.1590/S0004$\underline{27302013000300004}$

21. Nascimento ML, Rabello FH, Ohira M, Simoni $\mathrm{G}$, Cechinel E, Linhares RMM, et al. Programa de triagem neonatal para hipotireoidismo congênito de Santa Catarina, Brasil: avaliação etiológica no primeiro atendimento. Arq Bras Endocrinol Metabol. 2012;56(9):627-32. DOI: https://doi.org/10.1590/S0004$\underline{27302012000900005}$

22. Souza AS De, Jeunon A, Marinho F, Ferreira J. Etiologia do hipotireoidismo congênito e sua distribuição nas macrorregiões do estado de minas gerais. Rev Interdiscip Ciên Méd. 2018;2(31):22-8. 